ARTICLE

\title{
Multi-localization transport behaviour in bulk thermoelectric materials
}

Wenyu Zhao ${ }^{1, \star}$, Ping Wei ${ }^{1,2, \star}$, Qingjie Zhang ${ }^{1}$, Hua Peng ${ }^{3}$, Wanting Zhu' ${ }^{1}$ Dingguo Tang ${ }^{1}$, Jian Yu', Hongyu Zhou', Zhiyuan Liu', Xin Mu', Danqi He${ }^{1}$, Jichao $\mathrm{Li}^{3}$, Chunlei Wang ${ }^{3}$, Xinfeng Tang ${ }^{1} \&$ Jihui Yang ${ }^{2}$

Simultaneously optimizing electrical and thermal transport properties of bulk thermoelectric materials remains a key challenge due to the conflicting combination of material traits. Here, we have explored the electrical and thermal transport features of In-filled $\mathrm{CoSb}_{3}$ through X-ray absorption fine structure, X-ray photoemission spectra, transport measurement and theoretical calculation. The results provide evidence of three types of coexisting multilocalization transport behaviours in the material; these are heat-carrying phonon-localized resonant scattering, accelerated electron movement and increase in density of states near the Fermi level. The 5p-orbital hybridization between $\mathrm{In}$ and $\mathrm{Sb}$ is discovered in the In-filled $\mathrm{CoSb}_{3}$ compound, which results in a charge transfer from $\mathrm{Sb}$ to In and the enhancement of $p-d$ orbital hybridization between Co and Sb. Our work demonstrates that the electrical and thermal properties of filled skutterudite bulk thermoelectric materials can be simultaneously optimized through the three types of coexisting multi-localization transport behaviours in an independent way.

\footnotetext{
${ }^{1}$ State Key Laboratory of Advanced Technology for Materials Synthesis and Processing, Wuhan University of Technology, Wuhan 430070, China. ${ }^{2}$ Materials Science and Engineering Department, University of Washington, Seattle, Washington 98195, USA. ${ }^{3}$ School of Physics, State key Laboratory of Crystal Materials, Shandong University, Jinan 250100, China. * These authors contributed equally to this work. Correspondence and requests for materials should be addressed to Q.Z. (email: zhangqj@whut.edu.cn) or to J.Y. (email: jihuiy@uw.edu).
} 
T hermoelectric (TE) devices, which can directly convert heat into electricity and vice versa, have attracted considerable attention due to a variety of applications in heating, cooling, power generation and waste heat recovery ${ }^{1}$. Their conversion efficiency depends on the dimensionless figure of merit of TE materials defined as $Z T=\alpha^{2} \sigma T / \kappa$, where $T$ is the absolute temperature, $\sigma$ is the electrical conductivity, $\alpha$ is the Seebeck coefficient and $\kappa$ is the total thermal conductivity $\left(\kappa=\kappa_{\mathrm{E}}+\kappa_{\mathrm{L}}\right.$, where $\kappa_{\mathrm{E}}$ is the electronic contribution and $\kappa_{\mathrm{L}}$ the lattice contribution). Numerous efforts have been attempted to improve $Z T$ in the past two decades despite a compromise of $\kappa$ and $\alpha$ with $\sigma$ in TE materials ${ }^{2}$. To decrease $\kappa_{\mathrm{L}}$, various approaches used to enhance phonon scattering have taken advantage of nanoinclusion $^{3-6}$, alloying ${ }^{7}$, rattling filler ${ }^{8}$, quasi-ballistic transport nanoscale interfaces or nanopores ${ }^{9,10}$, liquid-like behaviour copper ions ${ }^{11}$ and anharmonic phonon coupling ${ }^{12}$. Meanwhile, a series of band structure engineering approaches such as high valley degeneracy ${ }^{13,14}$, peierls distortion ${ }^{15}$, electron energy filtering near the Fermi level ${ }^{16-18}$ and optimal bandwidth $^{19}$, have been employed to improve the electrical properties. Some important single-localization transport behaviours have been discovered in different TE materials. For example, interface scattering in $\mathrm{AgPb}_{m} \mathrm{SbTe}_{2+m}$ (ref. 4) and BiSbTe (ref. 6), and localized resonant scattering in filled $\mathrm{CoSb}_{3}$ (ref. 8) have remarkably enhanced phonon scattering and reduced $\kappa_{L}$; band convergence in $\mathrm{PbTe}_{1-x} \mathrm{Se}_{x}$ (ref. 13) and $\mathrm{Mg}_{2} \mathrm{Si}_{1-x} \mathrm{Sn}_{x}$ (ref. 14), charge density wave in $\mathrm{In}_{4} \mathrm{Se}_{3}$ (ref. 15) and electron resonant state in $\mathrm{PbTe}$ (ref. 16) have all led to an effective increase in $\alpha$. These single-localization transport behaviour, however, can only optimize a single physical parameter of electrical or thermal properties. So far, it remains a major challenge to simultaneously increase $\alpha$ and $\sigma$ while reducing $\kappa$, because no material has been found that shows multiple-localization transport behaviour.

Filled $\mathrm{CoSb}_{3}$ have been intensely pursued as an important TE material for intermediate-temperature power generation. The major progress in improving $Z T$ has made through decreasing $\kappa_{\mathrm{L}}$ by filling the icosahedron voids in $\mathrm{CoSb}_{3}$ with foreign atoms (for example, rare earths, alkali earths or alkali metals) to enhance heat-carrying phonon-localized resonant scattering via filler rattling ${ }^{8,20-29}$. Shi et al. $^{28}$ suggested that the electrical properties of multiple-filled $\mathrm{CoSb}_{3}$ could be optimized by adjusting the total filling fraction of fillers with different charge states. However, the tuning space of electrical properties is limited due to the conflicting relationship among $\alpha, \mathrm{n}$ and $\sigma$, as expressed in the following formulae:

$$
\begin{gathered}
\sigma=n e \mu_{\mathrm{H}} \\
\alpha=\frac{8 \pi^{2} k_{B}^{2} m^{*} T}{3 e h^{2}} \cdot \sqrt[3]{\left(\frac{\pi}{3 n}\right)^{2}}
\end{gathered}
$$

where $m^{*}$ is the carrier effective mass; $n$, the carrier concentration and $\mu_{\mathrm{H}}$, the carrier mobility. Recently, more and more experiments indicate that group III elements ( $\mathrm{Ga}$, In and $\mathrm{Tl}$ ) can remarkably improve $Z T$ of $\mathrm{CoSb}_{3}$ materials because of an almost perfect combination of low $\kappa_{\mathrm{L}}$, high $\sigma$ and large $\alpha^{30-38}$. However, it still remains unsettled how the group III elements synergistically adjust the electrical and thermal properties of $\mathrm{CoSb}_{3}$. The $\mathrm{Tl}$ filler rattling only explains the low $\kappa_{\mathrm{L}}$ of Tl-filled $\mathrm{CoSb}_{3}$ (refs 30,31). The dual-site occupancy at both the voids and $\mathrm{Sb}$ sites for $\mathrm{Ga}$ in $\mathrm{CoSb}_{3}$ is only responsible for low $\kappa_{\mathrm{L}}$ and $n^{32}$. $\mathrm{Up}$ to now, the doping behaviour of the In impurity in $\mathrm{CoSb}_{3}$ remains an ongoing debate $\mathrm{e}^{33-42}$.

In the following, we have explored the electrical and thermal transport features of In-filled $\mathrm{CoSb}_{3}$ through X-ray absorption fine structure (XAFS), X-ray photoemission spectra (XPS), transport measurement and theoretical calculation. Our data suggest that there are three types of coexisting multi-localization transport behaviours including heat-carrying phonon-localized resonant scattering, accelerated electron movement and increase in density of states (DOSs) near the Fermi level. Our work demonstrates that the electrical and thermal properties can be independently optimized through the three types of coexisting multi-localization transport behaviours.

\section{Results}

Filling behaviour of In impurity in $\mathrm{CoSb}_{3}$. We compare In $\mathrm{K}$ edge X-ray absorption near-edge structure (XANES) experimental spectra of quenched $\operatorname{In}_{0.2} \mathrm{Co}_{4} \mathrm{Sb}_{12}\left(\mathrm{Q}_{0.2}\right)$ and annealed $\operatorname{In}_{x} \mathrm{Co}_{4} \mathrm{Sb}_{12}(x=0.1,0.2$ and 0.25$)\left(\mathrm{A}_{x}\right)$ with those of InSb and In metal (Fig. 1). The In $K$-edge XANES spectrum of the In metal has five absorption peaks $A_{1}, B_{1}, C_{1}, D_{1}$ and $E_{1}$ centred at about 9 , $28,52,84$ and $128 \mathrm{eV}$, respectively, whereas that of InSb has four absorption peaks $\mathrm{A}_{2}, \mathrm{~B}_{2}, \mathrm{C}_{2}$ and $\mathrm{D}_{2}$ at about $11,35,64$ and $107 \mathrm{eV}$, respectively. The In K-edge XANES spectrum of $\mathrm{Q}_{0.2}$ has four absorption peaks with almost the same positions as those of $\mathrm{InSb}$, indicating the existence of InSb in $\mathrm{Q}_{0.2}$.

All the In $K$-edge XANES spectra of the $\mathrm{A}_{x}$ samples encompass five absorption peaks $A_{3}, B_{3}, C_{3}, D_{3}$ and $E_{3}$ with energy near 9,23 , 42,70 and $113 \mathrm{eV}$, respectively. The main peak $A_{3}$ has the same energy as that of $A_{1}$ for the In metal, but is $2 \mathrm{eV}$ lower than that of $\mathrm{A}_{2}$ for InSb. For the In $K$-edge XANES spectra, the main peaks $\mathrm{A}_{1}, \mathrm{~A}_{2}$ and $\mathrm{A}_{3}$ can be attributed to the $1 s \rightarrow 5 p$ transition. The energy discrepancy of $A_{2}$ and $A_{3}$ indicates that the chemical states of the In impurity are different between the $\mathrm{A}_{x}$ samples and InSb. It is worth noting that the absorption peaks $\mathrm{B}_{3}, \mathrm{C}_{3}, \mathrm{D}_{3}$ and $\mathrm{E}_{3}$ of all the $A_{x}$ samples are distinctly different in energy from $B_{1}, C_{1}$, $\mathrm{D}_{1}$ and $\mathrm{E}_{1}$ for the In metal, and from $\mathrm{B}_{2}, \mathrm{C}_{2}$ and $\mathrm{D}_{2}$ of InSb. Such significant differences undoubtedly show that the In impurities in the $\mathrm{A}_{x}$ samples are neither InSb nor the In metal. Accordingly, it

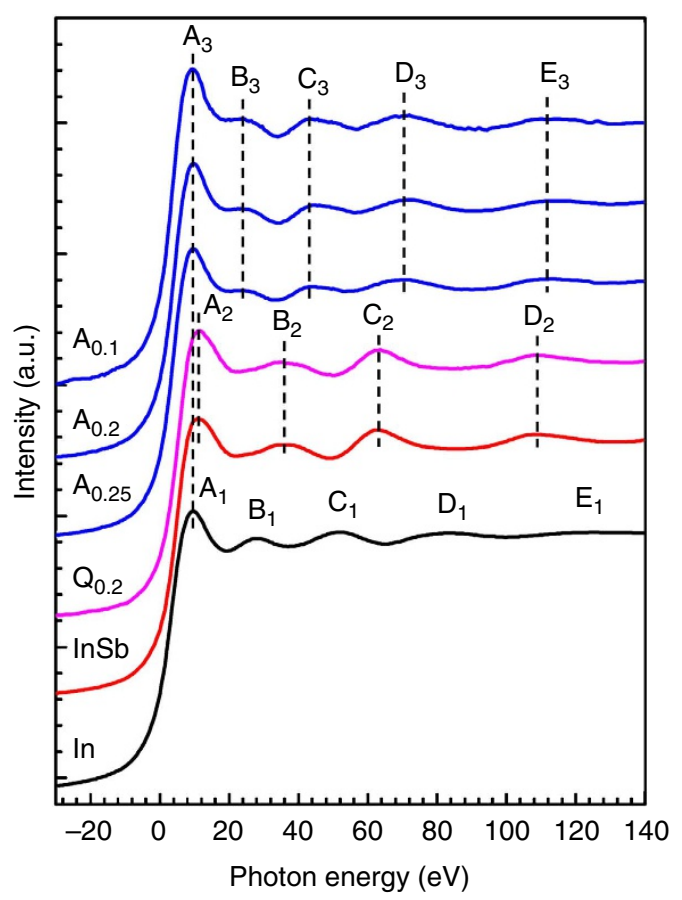

Figure 1 | The In $\boldsymbol{K}$-edge XANES experimental spectra. The quenched $\mathrm{In}_{0.2} \mathrm{CO}_{4} \mathrm{Sb}_{12}$ is symbolized with ' $\mathrm{Q}_{0.2}{ }^{\prime}$. The annealed $\ln _{x} \mathrm{Co}_{4} \mathrm{Sb}_{12}(x=0.1,0.2$ and 0.25 ) is symbolized with ' $\mathrm{A}_{x}$ '. The In $K$-edge XANES experimental spectra of InSb and In metals are plotted for comparison. Zero eV corresponds to the threshold of In K-edge $(27,940 \mathrm{eV})$. 
is highly plausible that the In impurities have been incoporated in the lattice of $\mathrm{CoSb}_{3}$ in all annealed samples, well consistent with the X-ray diffraction results (see Supplementary Fig. 1).

Because of the close electronegativity values among In (1.78), $\mathrm{Sb}$ (2.05) and Co (1.88), there exist four possible occupational sites for the In impurities in $\mathrm{CoSb}_{3}$, filling the icosahedron voids at the $2 a$ sites to form In-filled $\mathrm{CoSb}_{3}$, substituting for $\mathrm{Sb}$ at the



Figure 2 | The In K-edge XANES spectra of In impurity in $\mathrm{CoSb}_{\mathbf{3}}$.

(a) Filling $\mathrm{Sb}_{12}$ icosahedron voids at the $2 a$ sites, (b) substituting for $\mathrm{Sb}$ at the $24 \mathrm{~g}$ sites, (c) substituting for $\mathrm{Co}$ at the $8 \mathrm{c}$ sites and (d) simultaneously filling the icosahedron voids at the $2 a$ sites and substituting for $\mathrm{Sb}$ at the $24 \mathrm{~g}$ sites. The In K-edge XANES experimental spectra of $A_{0.25}$ are plotted for comparison. The XANES theoretical spectra are shown with red solid lines and symbolized as 'cal.'. The XANES experimental ones are shown with black circle lines and symbolized as 'exp.'
$24 \mathrm{~g}$ sites in the disordered $\mathrm{Sb}_{2} \mathrm{Co}_{2}$ tetrahedron to form In-doped $\mathrm{CoSb}_{3-s} \mathrm{In}_{s}$, substituting for Co at the $8 c$ sites in the irregular $\mathrm{Sb}_{6}$ octahedron to form In-doped $\mathrm{Co}_{1-r} \mathrm{In}_{r} \mathrm{Sb}_{3}$ or simultaneously filling the icosahedron voids at the $2 a$ sites and substituting for $\mathrm{Sb}$ at the $24 \mathrm{~g}$ sites to form $\left(\mathrm{In}_{\mathrm{VF}}\right)_{x 2 / 3} \mathrm{Co}_{4} \mathrm{Sb}_{12-x / 3}\left(\mathrm{In}_{\mathrm{Sb}}\right)_{x / 3}$ with charge-compensated compound defects ${ }^{33}$. The In $K$-edge XANES theoretical spectra of the In impurities at the $2 a, 24 g, 8 c$ and $2 a-$ $24 \mathrm{~g}$ sites in $\mathrm{CoSb}_{3}$ were calculated to identify which sites the In impurities occupy. The In $K$-edge XANES theoretical spectra (red solid lines symbolize 'cal.') of In-filled $\mathrm{CoSb}_{3}$ for the In impurities (a) filling icosahedron voids at the $2 a$ sites, (b) substituting for $\mathrm{Sb}$ at the $24 \mathrm{~g}$ sites, (c) substituting for $\mathrm{Co}$ at $8 c$ sites and (d) simultaneously filling the icosahedron voids at the $2 a$ sites and substituting for $\mathrm{Sb}$ at the $24 g$ sites are compared with the experimental spectrum (circle lines symbolize 'exp.') of the $\mathrm{A}_{0.25}$ sample (Fig. 2). It is clear that only the In K-edge XANES theoretical spectrum for filling icosahedron voids is in good agreement with the experimental data; the other three cases have large discrepencies between the theoretical spectra and the experimental ones (Fig. 2b-d). Therefore, the In K-edge XANES spectra unequivocally suggest that the In impurities stably fill the $\mathrm{Sb}_{12}$ icosahedron voids in $\mathrm{CoSb}_{3}$.

$5 p$-orbital hybridization between In and $\mathrm{Sb}$ and its effects. The total DOSs of $\mathrm{CoSb}_{3}$ and $\mathrm{In}_{0.125} \mathrm{Co}_{4} \mathrm{Sb}_{12}$, and partial DOS for Co, $\mathrm{Sb}$ and In atoms indicate that the total DOS of In-filled $\mathrm{In}_{0.125} \mathrm{Co}_{4} \mathrm{Sb}_{12}$ near valence band maximum (VBM) and conduction band minimum (CBM) mainly stem from Co $3 d$ electrons and Sb $5 p$ electrons (Fig. 3). It can be seen that there is an extra peak of the partial DOS of Co $3 d$ and Sb $5 p$ electrons near $0.31 \mathrm{eV}$ for $\mathrm{In}_{0.125} \mathrm{Co}_{4} \mathrm{Sb}_{12}$, which exactly corresponds to the highest peak of the partial DOS of In $5 p$ electrons. Particularly, the $\mathrm{DOS}_{\mathrm{Sb} 5 p} / \mathrm{DOS}_{\mathrm{Co} 3 d}$ ratio is decreased near VBM from 2.96 for $\mathrm{CoSb}_{3}$ to 2.08 for $\mathrm{In}_{0.125} \mathrm{Co}_{4} \mathrm{Sb}_{12}$ and increased near CBM from 0.20 for $\mathrm{CoSb}_{3}$ to 0.22 for $\mathrm{In}_{0.125} \mathrm{Co}_{4} \mathrm{Sb}_{12}$. This evolution suggests that the energy of Sb $5 p$ electrons and Co $3 d$ electrons becomes closer, and the $p-d$ orbital hybridization between $\mathrm{Co}$ and $\mathrm{Sb}$ has thus been enhanced in $\mathrm{In}_{0.125} \mathrm{Co}_{4} \mathrm{Sb}_{12}$. Experimentally, the XPS spectra of Co $2 p_{3 / 2}$ and $2 p_{1 / 2}$ core levels of In-filled $\mathrm{CoSb}_{3}$ are gradually shifted to higher binding energies (maximum up to
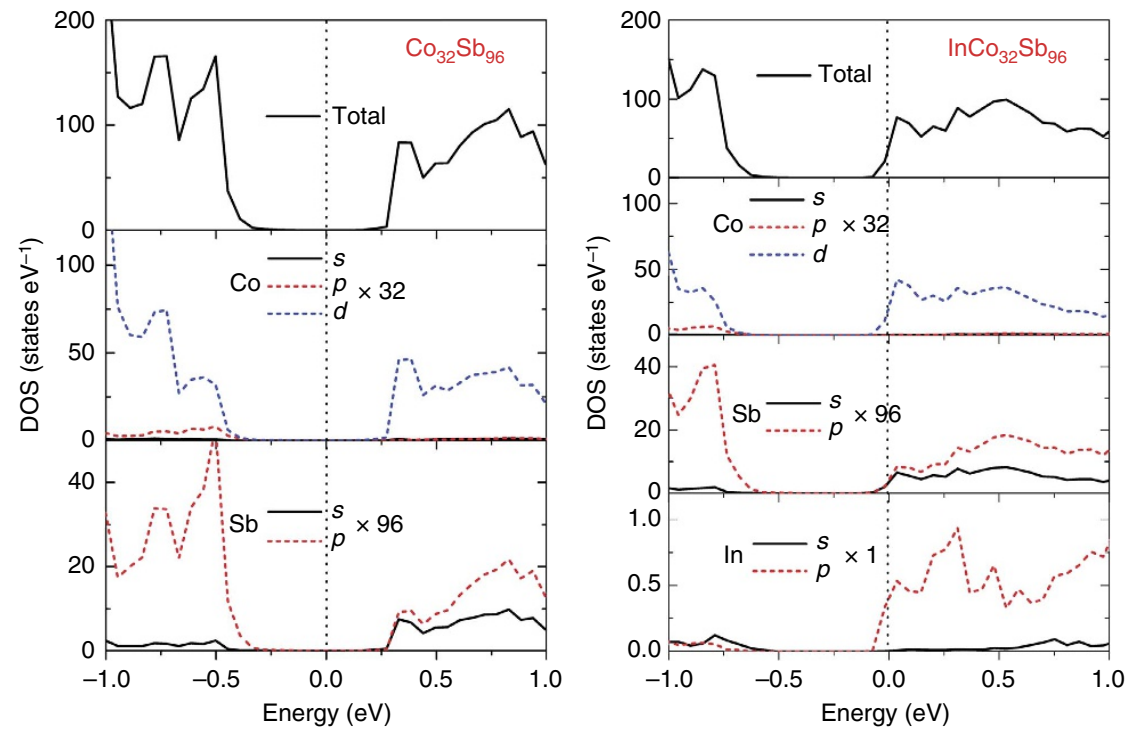

Figure 3 | Total DOS and partial DOS near VBM and CBM of $\mathbf{C o S} \mathbf{b}_{\mathbf{3}}$ and $\mathbf{I n}_{\mathbf{0 . 1 2 5}} \mathbf{C o}_{\mathbf{4}} \mathbf{S b}_{\mathbf{1 2}}$. The $2 \times 2 \times 2$ supercells were calculated using projectoraugmented wave method implemented in CASTEP package based on the density functional theory. 
$0.20 \mathrm{eV}$ ) as the filling fraction of the In filler increased (Fig. 4). The chemical shift is less than the energy resolution of XPS (about $0.47 \mathrm{eV}$ ) due to too low filling fraction of the In filler; however, the chemical shift can be repeated (see Supplementary Fig. 2) and thus may provide a plausible evidence of enhanced $p-d$ orbital hybridization between Co and Sb.

To clarify the origin of enhanced $p-d$ orbital hybridization between $\mathrm{Co}$ and $\mathrm{Sb}$ in In-filled $\mathrm{CoSb}_{3}$, the partial DOS of In atoms in the range of $-12 \sim 2 \mathrm{eV}$ have been analysed. We discover that the partial DOS of In $5 s$ electrons are distributed about $1.0 \mathrm{eV}$ below the Fermi level (see Supplementary Fig. 3). Therefore, all In $5 s$ electrons are confined at the deep locations of the valence band and have no contribution to $n$. Although there are a few In $5 p$ electrons below the Fermi level, the partial DOS of In $5 p$ electrons are mainly distributed above and near the Fermi level, suggesting that the In $5 p$ electrons are almost lost in Infilled $\mathrm{CoSb}_{3}$. The electronic states of the In impurity clearly show that the effective charge of the In filler is smaller than, but very close to, +1 . Therefore, the electronic configuration of the In filler is $5 s^{2} 4 d^{10} 5 p^{0}$, suggesting that the In filler may provide three unoccupied $5 p$ orbitals for a $5 p$-orbital hybridization between In



Figure 4 | XPS spectra of Co $2 p_{3 / 2}$ and $2 p_{1 / 2}$ core levels for CoSb $b_{3}$ and Infilled $\mathbf{C o S b}_{\mathbf{3}}$. Measurements were performed under the CAE mode with pass energy of $25 \mathrm{eV}$, step size of $0.05 \mathrm{eV}$ and 128 scans with a Thermo VG Multilab 2000 spectrometer.

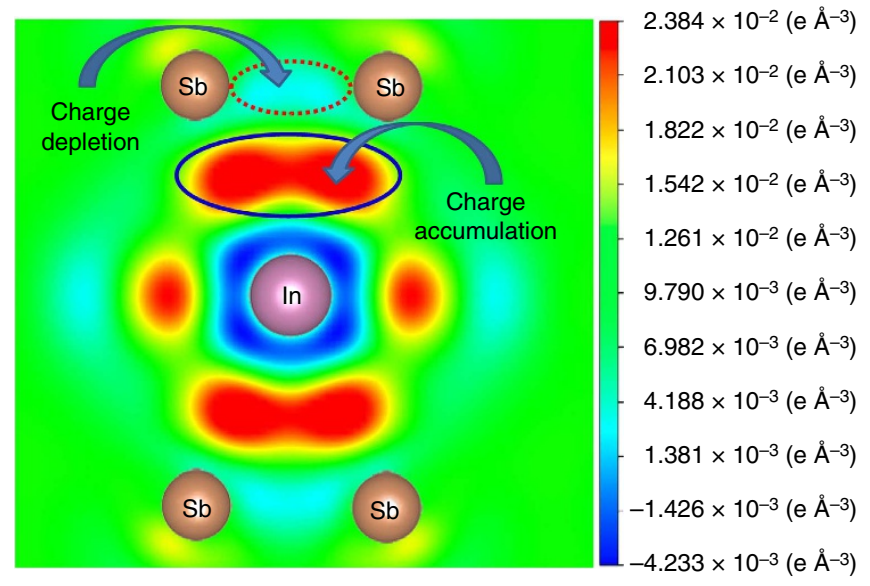

Figure 5 | Differential charge density of $\ln _{0.125} \mathrm{Co}_{4} \mathrm{Sb}_{12}$ projected on the (111) plane. The $2 \times 2 \times 2$ supercells were calculated using the projectoraugmented wave method implemented in CASTEP package based on the density functional theory. The differential charge density. delta

$p=p_{\ln 0325 b 96}-p_{\mathrm{C} 032 \mathrm{Sb} 96}-p_{\ln }$ and $\mathrm{Sb}$. This is corroborated by the differential charge density of $\mathrm{In}_{0.125} \mathrm{Co}_{4} \mathrm{Sb}_{12}$ projected on the (111) plane (Fig. 5) clearly showing the $5 p$-orbital hybridization between In and $\mathrm{Sb}$ in Infilled $\mathrm{CoSb}_{3}$. Therefore, the enhancement in $p-d$ orbital hybridization between $\mathrm{Co}$ and $\mathrm{Sb}$ in In-filled $\mathrm{CoSb}_{3}$ must originate from the $5 p$-orbital hybridization between In and $\mathrm{Sb}$. Note that the charge density decreases between $\mathrm{Sb}$ and $\mathrm{Sb}$ atoms while it increases between In and $\mathrm{Sb}$ atoms for In-filled icosahedron voids, indicating that the partial charges are transferred from $\mathrm{Sb}$ to In, which are in good agreement with our previous XPS results ${ }^{27}$. Namely, the $5 p$-orbital hybridization between In and $\mathrm{Sb}$ in In-filled $\mathrm{CoSb}_{3}$ can still cause a charge transfer from $\mathrm{Sb}$ to In and produce two types of atomic-scale electric fields near the In-filled $\mathrm{Sb}_{12}$ icosahedron, which are the atomic-scale electric fields with positive charge at the framework of In-filled $\mathrm{Sb}_{12}$ icosahedron and the atomic-scale electric fields with negative charge in the $\mathrm{Sb}_{12}$ icosahedron. Since the framework of $\mathrm{Sb}_{12}$ icosahedron acts as the passage of majority carriers (electrons) in In-filled $\mathrm{CoSb}_{3}$, the atomic-scale electric fields with positive charge at the framework of In-filled $\mathrm{Sb}_{12}$ icosahedron may accelerate electron movement.

In-Sb weak covalent bond and its effects. The extended XAFS (EXAFS) analysis reveals that the In-Sb bond length is about $3.35 \AA$ for the $\mathrm{A}_{0.2}$ sample (see Supplementary Fig. 4) and very close to the value (3.36 $\AA$ ) reported for $\mathrm{In}_{0.2} \mathrm{Co}_{4} \mathrm{Sb}_{12}$ (ref. 34), while it is only about $2.81 \AA$ for InSb ${ }^{43}$. The longer In-Sb bond indicates less orbital overlapping and weakened repulsion interaction between bonding and antibonding states of In-Sb bond in In-filled $\mathrm{CoSb}_{3}$. Therefore, the In-Sb bond between In filler and host framework of $\mathrm{Sb}_{12}$ icosahedron must be a weak covalent bond in In-filled $\mathrm{CoSb}_{3}$, further corroborating the lower energy of the main peak $A_{3}$ than that of $A_{2}$ (Fig. 1). Obviously, the In fillers can rattle inside the voids and cause heat-carrying phonon-localized resonant scattering, thereby remarkably reducing $\kappa_{\mathrm{L}}$.

The temperature dependences of $\kappa_{\mathrm{L}}$ values for $\mathrm{CoSb}_{3}$ and In-, $\mathrm{Ba}$ - and Ga-filled $\mathrm{CoSb}_{3}$ (Fig. 6) show that $\kappa_{\mathrm{L}}$ value at $300 \mathrm{~K}$ is only about $5.16 \mathrm{~W} \mathrm{~m}^{-1} \mathrm{~K}^{-1}$ for $\mathrm{In}_{0.08} \mathrm{Co}_{4} \mathrm{Sb}_{12}$ and $3.75 \mathrm{~W} \mathrm{~m}^{-1}$ $\mathrm{K}^{-1}$ for $\mathrm{In}_{0.18} \mathrm{Co}_{4} \mathrm{Sb}_{12}$ while more than $10 \mathrm{~W} \mathrm{~m}^{-1} \mathrm{~K}^{-1}$ for $\mathrm{CoSb}_{3}$. The $\kappa_{\mathrm{L}}$ values of $\mathrm{In}_{0.08} \mathrm{Co}_{4} \mathrm{Sb}_{12}$ are smaller than those of $\mathrm{Ba}_{0.09} \mathrm{Co}_{4} \mathrm{Sb}_{12}$ in the range of $300-650 \mathrm{~K}$, suggesting that the In filler is more effective in reducing $\kappa_{\mathrm{L}}$ than $\mathrm{Ba}$ at a comparable filling fraction. The $\kappa_{\mathrm{L}}$ values of $\mathrm{In}_{0.08} \mathrm{Co}_{4} \mathrm{Sb}_{12}$, however, are significantly greater than those of $\mathrm{Ga}_{0.09} \mathrm{Co}_{4} \mathrm{Sb}_{12}$ in the range of

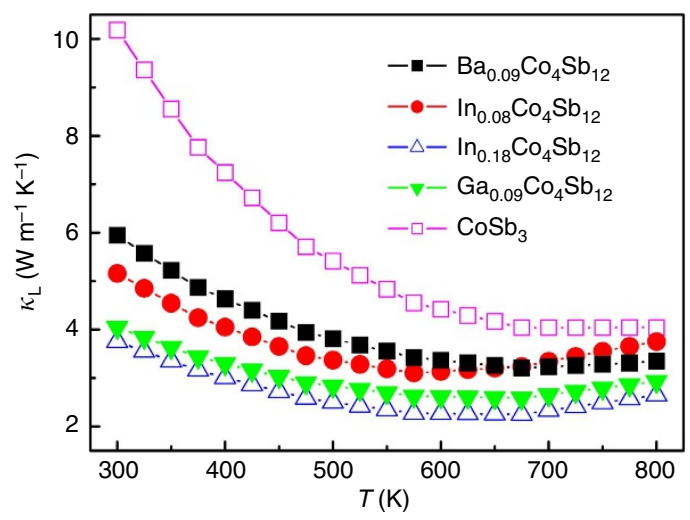

Figure 6 | Temperature dependences of lattice thermal conductivity in the range of $\mathbf{3 0 0 - 8 0 0 ~ K}$. The data of $\mathrm{CoSb}_{3}$ are plotted for comparison with those of In-, Ba-, and Ga-filled $\mathrm{CoSb}_{3}$. 
$300-800 \mathrm{~K}$, suggesting different doping behaviour in $\mathrm{CoSb}_{3}$ between In and $\mathrm{Ga}$. The lower $\kappa_{\mathrm{L}}$ values of $\mathrm{Ga}_{0.09} \mathrm{Co}_{4} \mathrm{Sb}_{12}$ are due to the additional defect scattering induced by the $\mathrm{Sb}$ substitutional $\mathrm{Ga}$, because $\mathrm{Ga}$ impurties in $\mathrm{CoSb}_{3}$ were thought to simultaneously occupy both the icosahedron voids and the $\mathrm{Sb}$ sites $^{32}$.

\section{Discussion}

The $n$ values of $\operatorname{In}_{0.18} \mathrm{Co}_{4} \mathrm{Sb}_{12}$ are almost the same as those of $\mathrm{Ba}_{0.09} \mathrm{Co}_{4} \mathrm{Sb}_{12}$ in the range of $100-300 \mathrm{~K}$ (Fig. 7), clearly indicating that the In filler provides one electron and is univalent $\left(\mathrm{In}^{+}\right)$in In-filled $\mathrm{CoSb}_{3}$ because the $\mathrm{Ba}$ filler provides two electrons in Ba-filled $\mathrm{CoSb}_{3}$. The electronic structure of the In filler described above not only is the physical mechanism of low $n$ for In-filled $\mathrm{CoSb}_{3}$, but also may reasonably explain why the $n$ values of $\mathrm{In}_{0.18} \mathrm{Co}_{4} \mathrm{Sb}_{12}$ and $\mathrm{Ba}_{0.09} \mathrm{Co}_{4} \mathrm{Sb}_{12}$ are very close in the range of $100-300 \mathrm{~K}$. At the same time, the charge transfer from $\mathrm{Sb}$ to In in In-filled $\mathrm{CoSb}_{3}$ must produce the same amount of atomic-scale electric fields with positive charge at the framework of In-filled $\mathrm{Sb}_{12}$ icosahedron; therefore, the major carriers (electrons) nearby the In-filled $\mathrm{Sb}_{12}$ icosahedron are not only partially annihilated but also accelerated because of the attraction by the atomic-scale electric fields with positive charge. These multi-functional local transport effects may explain that the Infilled $\mathrm{CoSb}_{3}$ has higher $\mu_{\mathrm{H}}$ than those of Ba-filled and Ga-filled $\mathrm{CoSb}_{3}$ in the range of $100-300 \mathrm{~K}$ under comparable $n$ values (Fig. 8). As a result, the In-filled $\mathrm{CoSb}_{3}$ have higher $\sigma$ than $\mathrm{Ba}$ and Ga-filled $\mathrm{CoSb}_{3}$ in the range of $150-800 \mathrm{~K}$, although their $n$ values are very close (Fig. 9), which can be attributed to an increase in $\mu_{\mathrm{H}}$ induced by accelerated electron movement nearby the In-filled $\mathrm{Sb}_{12}$ icosahedron. Compared with $\mathrm{Ba}$ - and In-filled $\mathrm{CoSb}_{3}$, the lower $\mu_{\mathrm{H}}$ of Ga-filled $\mathrm{CoSb}_{3}$ in the range of $10-100 \mathrm{~K}$ may be reasonably explained by the dual-site occupancy of $\mathrm{Ga}$ impurity in $\mathrm{CoSb}_{3}$ (ref. 32). In addition, the $\mu_{\mathrm{H}}$ values of Ba- and In-filled $\mathrm{CoSb}_{3}$ share similar temperature dependence in the range of $10-300 \mathrm{~K}$ (Fig. 8), implying that the electron scattering mechanisms are the same for both cases and there is thus no case of In occupying at the Sb sites. This is well consistent with the XANES results as shown in Fig. 2 .

The enhancement of the $p-d$ orbital hybridization between Co and $\mathrm{Sb}$ induced by the In filler still provides a more reasonable



Figure 7 | Temperature dependences of carrier concentration in the range of 10-300 K. The inset shows the temperature dependences of carrier concentration of $\mathrm{In}-, \mathrm{Ba}-$, and Ga-filled $\mathrm{CoSb}_{3}$ in the range of 100-300K explanation for the band structure of $\operatorname{In}_{0.125} \mathrm{Co}_{4} \mathrm{Sb}_{12}$. Compared with $\mathrm{CoSb}_{3}$ (see Supplementary Fig. 5), the Fermi level of $\mathrm{In}_{0.125} \mathrm{Co}_{4} \mathrm{Sb}_{12}$ is migrated into conduction bands and the energy gap between the Fermi level and CBM at $H, N$ and $P$ points with high symmetry is significantly decreased from $0.45 \sim 0.35 \mathrm{eV}$ for $\mathrm{CoSb}_{3}$ to $0.12 \sim 0.03 \mathrm{eV}$ for $\mathrm{In}_{0.125} \mathrm{Co}_{4} \mathrm{Sb}_{12}$. As a result, the DOS of VBM is significantly decreased while the DOS of CBM is remarkably increased. Namely, there is an asymmetric distribution of DOS near the Fermi level of In-filled $\mathrm{CoSb}_{3}$ beneficial to obtaining a large $\alpha$. Such a DOS asymmetric distribution may very well explain why the absolute $\alpha$ values of In-filled $\mathrm{CoSb}_{3}$

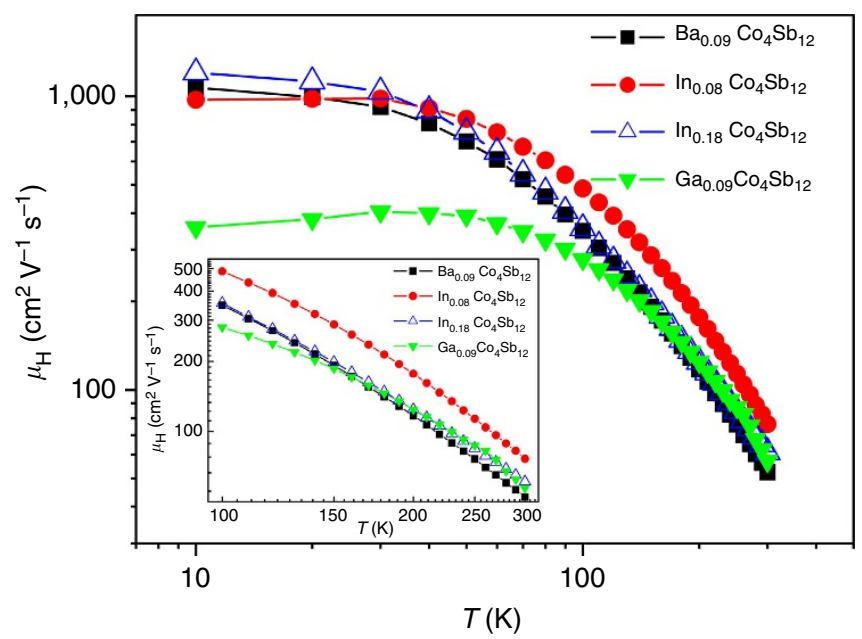

Figure 8 | Temperature dependences of Hall mobility in the range of 10-300 K. The inset shows the temperature dependences of Hall mobility of In-, Ba-, and Ga-filled $\mathrm{CoSb}_{3}$ in the range of $100-300 \mathrm{~K}$.
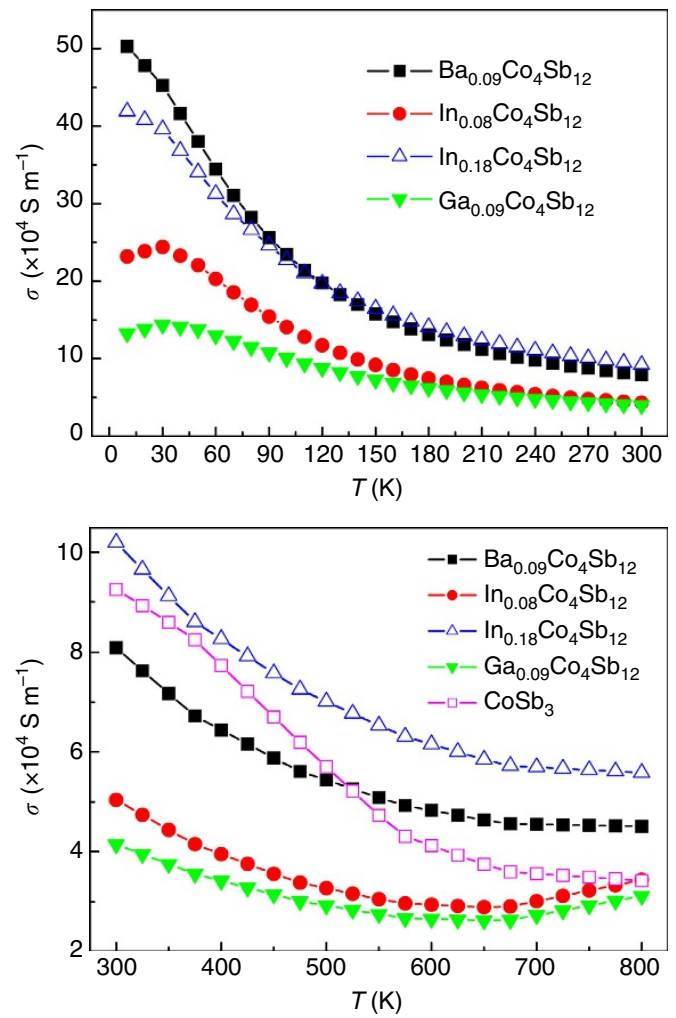

Figure 9 | Temperature dependences of electrical conductivity in the range of $\mathbf{1 0 - 8 0 0 ~ K}$. The data of $\mathrm{CoSb}_{3}$ in the range of 300-800K are plotted for comparison with those of In-, Ba-, and Ga-filled CoSb ${ }_{3}$. 


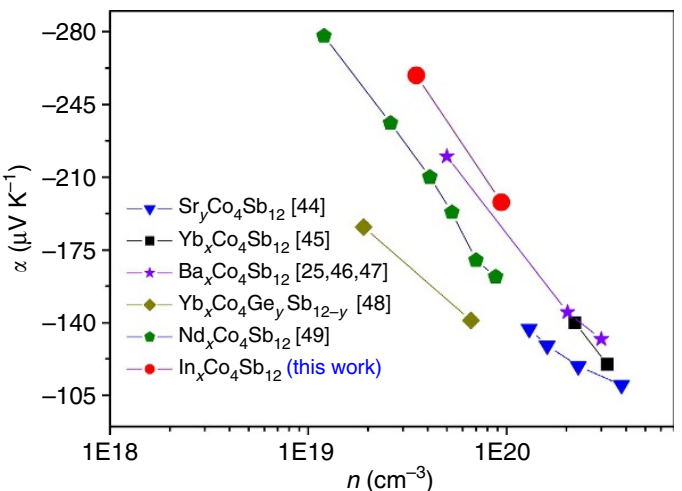

Figure 10 | Carrier concentration dependences of the Seebeck coefficient of $\boldsymbol{n}$-type filled $\mathrm{CoSb}_{\mathbf{3}}$ at room temperature. Here, compare the data of $\mathrm{Sr}-, \mathrm{Yb}-, \mathrm{Ba}-, \mathrm{Nd}-$, and In-filled $\mathrm{CoSb}_{3}$ with $\mathrm{n}$ on the order of $10^{19} \sim 10^{20} \mathrm{~cm}^{-3}$.

are higher than those of $n$-type $\mathrm{Ba}-, \mathrm{Sr}-, \mathrm{Yb}$ - and $\mathrm{Nd}$-filled $\mathrm{CoSb}_{3}$, with similar $n$ on the order of $10^{19} \mathrm{~cm}^{-3}$ at room temperature $25,44-49$, as shown in Fig. 10 . The $\alpha$ values at $300 \mathrm{~K}$ reached $259 \mu \mathrm{V} \mathrm{K}^{-1}$ for $\operatorname{In}_{0.08} \mathrm{Co}_{4} \mathrm{Sb}_{12}$ with $3.5 \times 10^{19} \mathrm{~cm}^{-3}$ and $198 \mu \mathrm{V} \mathrm{K}^{-1}$ for $\mathrm{In}_{0.18} \mathrm{Co}_{4} \mathrm{Sb}_{12}$ with $9.4 \times 10^{19} \mathrm{~cm}^{-3}$. Obviously, the large $\alpha$ values of In-filled $\mathrm{CoSb}_{3}$ originate from the increase in DOS of CBM near the Fermi level due to enhanced $p-d$ orbital hybridization between $\mathrm{Co}$ and $\mathrm{Sb}$ induced by the In filler.

Therefore, the perfect combination of low $\kappa_{\mathrm{L}}$, high $\sigma$ and large $\alpha$ for In-filled $\mathrm{CoSb}_{3}$ originates from the following physical and chemical mechanisms. First, the low $\kappa_{\mathrm{L}}$ is due to the heat-carrying phonon-localized resonant scattering induced by In-filler rattling. Second, the high $\sigma$ is attributed to the accelerated electron movement induced by the charge transfer from Sb to In. Third, the large $\alpha$ benefits from the increase in DOS of CBM near the Fermi level induced by the enhanced $p-d$ orbital hybridization between $\mathrm{Co}$ and $\mathrm{Sb}$. The $5 p$-orbital hybridization between In and $\mathrm{Sb}$ in In-filled $\mathrm{CoSb}_{3}$ can cause a charge transfer from $\mathrm{Sb}$ to In and the enhancement of $p-d$ orbital hybridization between Co and $\mathrm{Sb}$. The fundamental origin of low $n$ of In-filled $\mathrm{CoSb}_{3}$ is that all $5 s$ electrons of the In filler are confined at the deep locations of the valence band. The low $n$ and asymmetric distribution of DOS near the Fermi level provide a favourable condition for adjusting $\sigma$ and $\alpha$ of In-filled $\mathrm{CoSb}_{3}$ in an independent way.

\begin{abstract}
Methods
Synthesis and characterization. In-filled $\operatorname{In}_{x} \mathrm{Co}_{4} \mathrm{Sb}_{12}(x=0.1,0.2$ and 0.25$)$ bulk materials were prepared by a combination of melting, annealing and spark plasma sintering reported elsewhere ${ }^{25}$. Another three bulk materials $\left(\mathrm{CoSb}_{3}, \mathrm{Ba}_{0.1} \mathrm{Co}_{4} \mathrm{Sb}_{12}\right.$ and $\mathrm{Ga}_{0.1} \mathrm{Co}_{4} \mathrm{Sb}_{12}$ ) were prepared with the same method for comparsion. X-ray diffraction (PANalytical X' Pert PRO) and scanning electron microscope analysis confirmed that all the annealed samples $\operatorname{In}_{x} \mathrm{Co}_{4} \mathrm{Sb}_{12}(x=0.1,0.2$ and 0.25$)$ were composed of single-phase skutterudite, while all the quenched samples consisted of $\mathrm{Sb}, \mathrm{CoSb}, \mathrm{CoSb}_{2}$ and InSb. Chemical compositions of all the bulk materials were determined by electron probe microanalysis (EPMA, JXA-8230). XANES and EXAFS of quenched $\mathrm{In}_{0.2} \mathrm{Co}_{4} \mathrm{Sb}_{12}$ and annealed $\mathrm{In}_{x} \mathrm{Co}_{4} \mathrm{Sb}_{12}$ samples were measured under a working voltage of $3.5 \mathrm{GeV}$ and a working current of $300 \mathrm{~mA}$ at BL14W1 beamline in the Shanghai Synchrotron Radiation Facility (SSRF). A Si (311) double-crystal monochromater with energy resolution of $0.5 \times 10^{-4} \mathrm{eV} @ 10 \mathrm{keV}$ was employed to measure In $K$-edge spectra. All XANES and EXAFS spectra were measured three times to ensure reproducibility. The In $K$-edge XANES spectra of InSb and the In metal were also recorded for comparison. XPS of Co $2 p_{3 / 2}$ and $2 p_{1 /}$ ${ }_{2}$ core levels were recorded at pass energy of of $25 \mathrm{eV}$, step size of $0.05 \mathrm{eV}$ and 128 scans with Thermo VG Multilab 2000 spectrometer.
\end{abstract}

Transport measurement. The $\sigma$ and $\alpha$ values were measured with the standard four-probe method (UlvacRiko: ZEM-3) in Ar atmosphere. The $\kappa$ was calculated using the equation $\kappa=D \rho C_{\mathrm{p}}$, where $C_{\mathrm{p}}$ is the specific heat capacity, $\rho$ the bulk density and $D$ the thermal diffusion coefficient. $D$ was measured by a laser flash technique (Netzsch LFA 427) in a flowing Ar atmosphere, $C_{\mathrm{p}}$ with a TA Q20 differential scanning calorimeter and $\rho$ by Archimedes method. $\kappa_{\mathrm{L}}$ was obtained by subtracting the electrical contribution from $\kappa$ using the equation $\kappa_{\mathrm{L}}=\kappa-\kappa_{\mathrm{E}} \cdot \kappa_{\mathrm{E}}$ is expressed by the Wiedemann-Franz $\kappa_{\mathrm{E}}=\sigma L T$, where $L$ is the Lorenz number. Uncertainties are $\pm 5-7 \%$ for $\sigma$ and $\kappa_{\mathrm{L}}$, and $\pm 5 \%$ for $\alpha$. The $n$ and $\mu_{\mathrm{H}}$ were measured under 10-300 K with Quantum Design PPMS.

Theoretical calculation. The $K$-edge XANES theoretical spectra of In impurity at four kinds of crystallographic sites $(2 a, 24 g, 8 c$ and $2 a-24 g)$ in $\mathrm{CoSb}_{3}$ were calculated with the self-consistent multiple-scattering theory based on real-space clusters implemented in FEFF9 package ${ }^{50}$. The In $K$-edge EXAFS experimental spectra were first normalized and background subtracted to obtain the $k$-weighted spectra, and then Fourier transformed to obtain the length of the In-Sb bond. The DOSs, band structure and differential charge densities projected on the (111) plane of $\mathrm{CoSb}_{3}$ and $\mathrm{In}_{0.125} \mathrm{Co}_{4} \mathrm{Sb}_{12}$ using a $2 \times 2 \times 2$ supercell were calculated using a projector-augmented wave method implemented in CASTEP package based on the density functional theory ${ }^{51}$. Lattice relaxation and structural optimization were carried out through total energy calculations.

\section{References}

1. Bell, L. E. Cooling, heating, generating power, and recovering waste heat with thermoelectric systems. Science 321, 1457-1461 (2008).

2. Snyder, G. J. \& Toberer, E. S. Complex thermoelectric materials. Nat. Mater. 7, 105-114 (2008).

3. Biswas, K. et al. High-performance bulk thermoelectrics with all-scale hierarchical architectures. Nature 489, 414-418 (2012).

4. Hsu, K. F. et al. Cubic $\mathrm{AgPb}_{m} \mathrm{SbTe}_{2+m}$ : Bulk thermoelectric materials with high figure of merit. Science 303, 818-821 (2004).

5. Dresselhaus, M. S. et al. New directions for low-dimensional thermoelectric materials. Adv. Mater. 19, 1043-1053 (2007).

6. Poudel, B. et al. High-thermoelectric performance of nanostructured bismuth antimony telluride bulk alloys. Science 320, 634-638 (2008).

7. Morelli, D. T., Jovovic, V. \& Heremans, J. P. Intrinsically minimal thermal conductivity in cubic $\mathrm{I}-\mathrm{V}-\mathrm{VI}_{2}$ semiconductors $\mathrm{AgSbTe}_{2}$ and $\mathrm{AgBiSe} e_{2}$. Phys. Rev. Lett. 101, 035901-1-035901-4 (2008).

8. Sales, B. C., Mandrus, D. \& Williams, R. K. Filled skutteruditeantimonides: a new class of thermoelectric materials. Science 272, 1325-1328 (1996).

9. Siemens, M. E. et al. Quasi-ballistic thermal transport from nanoscale interfaces observed using ultrafast coherent soft X-ray beams. Nat. Mater. 9, 26-30 (2010).

10. Zhao, W. Y. et al. Enhanced thermoelectric performance via randomly arranged nanopores: excellent transport properties of $\mathrm{YbZn}_{2} \mathrm{Sb}_{2}$ nanoporous materials. Acta Mater. 60, 1741-1746 (2012).

11. Liu, H. L. et al. Copper ion liquid-like thermoelectric. Nat. Mater. 11, 422-425 (2012).

12. Delaire, O. et al. Giant anharmonic phonon scattering in PbTe. Nat. Mater. 10, 614-619 (2011).

13. Pei, Y. Z. et al. Convergence of electronic bands for high performance bulk thermoelectric. Nature 473, 66-69 (2011).

14. Liu, W. et al. Convergence of conduction bands as a means of enhancing thermoelectric performance of $\mathrm{n}$-type $\mathrm{Mg}_{2} \mathrm{Si}_{1-\mathrm{x}} \mathrm{Sn}_{\mathrm{x}}$ solid solutions. Phys. Rev. Lett. 108, 166601-166605 (2012).

15. Rhyee, J. S. et al. Peierls distortion as a route to high thermoelectric performance in $\mathrm{In}_{4} \mathrm{Se}_{3}$-delta crystals. Nature 459, 965-968 (2009).

16. Heremans, J. P. et al. Enhancement of thermoelectric efficiency in $\mathrm{PbTe}$ by distortion of the electronic density of states. Science 321, 554-557 (2008).

17. Ahmad, S., Hoang, K. \& Mahanti, S. D. Ab Initio study of deep defect states in narrow band-gap semiconductors: Group III impurities in PbTe. Phys. Rev. Lett. 96, 056403-1-056403-4 (2006).

18. Lee, J.-H., Wu, J. \& Grossman, J. C. Enhancing the thermoelectric power factor with highly mismatched isoelectronic doping. Phys. Rev. Lett. 104, 016602-1-066602-4 (2010).

19. Zhou, J., Yang, R. G., Chen, G. \& Dresselhaus, M. S. Optimal bandwidth for high efficiency thermoelectric. Phys. Rev. Lett. 107, 226601-1-226601-5 (2011).

20. Nolas, G. S., Cohn, J. L. \& Slack, G. A. Effect of partial void filling on the lattice thermal conductivity of skutterudites. Phys. Rev. B 58, 164-170 (1998).

21. Uher, C. in Recent Trends in Thermoelectric Materials Research I, Semiconductors and Semimetals (ed. Tritt, T. M.) Vol. 69 139-253 (Academic Press, 2001).

22. Nolas, G. S., Kaeser, M., Littleton, R. T. \& Tritt, T. M. High figure of merit in partially filled ytterbium skutterudite materials. Appl. Phys. Lett. 77, 1855-1857 (2000).

23. Morelli, D. T., Meisner, G. P., Chen, B. X., Hu, S. Q. \& Uher, C. Cerium filling and doping of cobalt triantimonide. Phys. Rev. B 56, 7376-7383 (1997).

24. Chen, L. D. et al. Anomalous barium filling fraction and $n$-type thermoelectric performance of $\mathrm{Ba}_{y} \mathrm{Co}_{4} \mathrm{Sb}_{12}$. J. Appl. Phys. 90, 1864-1868 (2001). 
25. Zhao, W. Y. et al. Synthesis and high temperature transport properties of barium and indium double-filled skutterudites $\mathrm{Ba}_{x} \mathrm{In}_{y} \mathrm{Co}_{4} \mathrm{Sb}_{12-z}$. J. Appl. Phys. 102, 113708-1-113708-6 (2007).

26. Pei, Y. Z. et al. Improving thermoelectric performance of caged compounds through light-element filling. Appl. Phys. Lett. 95, 042101-1-042101-3 (2009).

27. Zhao, W. Y. et al. Enhanced thermoelectric performance in barium and indium double-filled skutterudite bulk materials via orbital hybridization induced by indium filler. J. Am. Chem. Soc. 131, 3713-3720 (2009).

28. Shi, X. et al. Multiple-filled skutterudites: high thermoelectric figure of merit through separately optimizing electrical and thermal transports. J. Am. Chem. Soc. 133, 7837-7846 (2011)

29. Mei, Z. G., Yang, J., Pei, Y. Z., Zhang, W. \& Chen, L. D. Alkali-metal-filled $\mathrm{CoSb}_{3}$ skutterudites as thermoelectric materials: theoretical study. Phys. Rev. B 77, 045202-1-045202-8 (2008).

30. Sales, B. C., Chakoumakos, B. C. \& Mandrus, D. Thermoelectric properties of thallium-filled skutterudites. Phys. Rev. B 61, 2475-2481 (2000).

31. Hermann, R. P. et al. Einstein oscillators in thallium filled antimony skutterudites. Phys. Rev. Lett. 90, 135505-1-135505-4 (2003).

32. Qiu, Y. T. et al. Charge-compensated compound defects in Ga-containing thermoelectric skutterudites. Adv. Funct. Mater. 23, 3194-3203 (2013).

33. Tang, Y. L. et al. Phase diagram of In-Co-Sb system and thermoelectric properties of In-containing skutterudites. Energy Environ. Sci. 7, 812-819 (2014).

34. He, T., Chen, J. Z., Rosenfeld, H. D. \& Subramanian, M. A. Thermoelectric properties of indium-filled skutterudites. Chem. Mater. 18, 759-762 (2006).

35. Harnwunggmoung, A. et al. Enhancement of thermoelectric properties of $\mathrm{CoSb}_{3}$-based skutterudites by double filling of $\mathrm{Tl}$ and In. J. Appl. Phys. 112, 043509-1-043509-6 (2012).

36. Wang, L., Cai, K. F., Wang, Y. Y., Li, H. \& Wang, H. F. Thermoelectric properties of indium-filled skutterudites prepared by combining solvothermal synthesis and melting. Appl. Phys. A 97, 841-845 (2009).

37. Wei, P. et al. Excellent performance stability of Ba and In double-filled skutterudite thermoelectric materials. Acta Mater. 59, 3244-3254 (2011).

38. Yu, J. et al. Effect of In impurity on thermoelectric properties of Ba and In double-filled n-type skutterudite materials. J. Electron. Mater. 41, 1395-1400 (2012).

39. Peng, J. Y. et al. High temperature thermoelectric properties of double-filled $\mathrm{In}_{x} \mathrm{Yb}_{y} \mathrm{Co}_{4} \mathrm{Sb}_{12}$ skutterudites. J. Appl. Phys. 105, 084907-1-084907-5 (2009).

40. Grytsiv, A., Rogl, P., Michor, H., Bauer, E. \& Giester, G. $\operatorname{In}_{\mathrm{y}} \mathrm{Co}_{4} \mathrm{Sb}_{12}$ skutterudite: Phase equilibria and crystal structure. J. Electron. Mater. 42, 2940-2952 (2013)

41. Eilertsen, J., Rouvimov, S. \& Subramanian, M. A. Rattler-seeded InSb nanoinclusions from metastable indium-filled $\mathrm{In}_{0.1} \mathrm{Co}_{4} \mathrm{Sb}_{12}$ skutterudites for high-performance thermoelectric. Acta Mater. 60, 2178-2785 (2012).

42. Li, H., Tang, X. F., Zhang, Q. J. \& Uher, C. High performance $\operatorname{In}_{x} \mathrm{Ce}_{y} \mathrm{Co}_{4} \mathrm{Sb}_{12}$ thermoelectric materials with in situ forming nanostructured InSb phase. Appl. Phys. Lett. 94, 102114-1-102114-3 (2009).

43. Chen, D. L., Li, C. S., Zhu, Z. G., Fan, J. W. \& Wei, S. Q. Interface effect of InSb quantum dots embedded in $\mathrm{SiO}_{2}$ matrix. Phys. Rev. B 72, 075341-1-075341-7 (2005).

44. Zhao, X. Y. et al. Synthesis and thermoelectric properties of Sr-filled skutterudite $\mathrm{Sr}_{y} \mathrm{Co}_{4} \mathrm{Sb}_{12}$. J. Appl. Phys. 99, 053711-1-053711-4 (2006).

45. Li, H., Tang, X. F., Su, X. L., Zhang, Q. J. \& Uher, C. Nanostructured bulk $\mathrm{Yb}_{x} \mathrm{Co}_{4} \mathrm{Sb}_{12}$ with high thermoelectric performance prepared by the rapid solidification method. J. Phys. D Appl. Phys. 42, 145409-1-145409-9 (2009).

46. Bai, S. Q. et al. Enhanced thermoelectric performance of dual-element-filled skutterudites $\mathrm{Ba}_{x} \mathrm{Ce}_{y} \mathrm{Co}_{4} \mathrm{Sb}_{12}$. Acta Mater. 57, 3135-3139 (2009).

47. Shi, X. et al. Low thermal conductivity and high thermoelectric figure of merit in n-type $\mathrm{Ba}_{x} \mathrm{Yb}_{y} \mathrm{Co}_{4} \mathrm{Sb}_{12}$ double-filled skutterudites. Appl. Phys. Lett. 92, 182101-1-182101-3 (2008).
48. Lamberton, Jr. G. A., Tedstrom, R. H., Tritt, T. M. \& Nolas, G. S. Thermoelectric properties of Yb-filled Ge-compensated $\mathrm{CoSb}_{3}$ skutterudite materials. J. Appl. Phys. 97, 113715-1-113715-5 (2005).

49. Kuznetsov, V. L., Kuznetsova, L. A. \& Rowe, D. M. Effect of partial void filling on the transport properties of $\mathrm{Nd}_{x} \mathrm{Co}_{4} \mathrm{Sb}_{12}$ skutterudites. J. Phys Condens. Matter. 15, 5035-5048 (2003).

50. Rehr, J. J. \& Albers, R. C. Theoretical approaches to X-ray absorption fine structure. Rev. Mod. Phys. 72, 621-654 (2000).

51. Payne, M. C., Teter, M. P., Allan, D. C., Arias, T. A. \& Joannopoulos, J. D. Iterative minimization techniques for $a b$ initio total-energy calculations: molecular dynamics and conjugate gradients. Rev. Mod. Phys. 64, 1045-1097 (1992).

\section{Acknowledgements}

This work was supported by the National Basic Research Program of China (973 program) under Project No. 2013CB632505, and National Natural Science Foundation of China (Nos 50930004, 50972114 and 11274248). XANES and EXAFS measurements were performed at BL14W1 beamline in Shanghai Synchrotron Radiation Facility. XRD, SEM and EPMA experiments were performed at the Center for Materials Research and Testing of Wuhan University of Technology. Hall measurements were performed at US General Motors Corp. and State Key Lab of Advanced Technology for Materials Synthesis and Processing of Wuhan University of Technology. X-ray photoemission spectra were recorded at Key Laboratory of Catalysis and Materials Science of the State Ethnic Affair Commission \& Ministry of Education of South-Central University for Nationalities and School of Physics and Technology of Wuhan University. We thank Dr Zhang and Professor Jiang for their help in XANES and EXAFS measurements. We also thank Professor G.S. Shao from the University of Bolton for the valuable discussions on XANES and EXAFS analysis; thanks to Professor L.M. Mei from Shandong University, Professor J. Shi and Professor H.J. Liu from Wuhan University and Professor X.M. Min from Wuhan University of Technology for their valuable discussions on the electronic structure of In-filled $\mathrm{CoSb}_{3}$.

\section{Author contributions}

W.Z., P.W. and Q.Z. designed and carried out XANES and EXAFS experiments. P.W., W.Z., D.T., J.Y., H.Z. and Z.L. synthesized the samples and carried out the thermoelectric properties measurements. H.P., J.L. and C.W. performed the electron structure calculations. W.Z., P.W., W.Z., X.T. and J.Y. performed the Hall measurements. W.Z. and D.T. performed the XPS measurements. W.Z., P.W., Q.Z and J.Y. conceived the experiments, analysed the results and wrote and edited the manuscript. All authors read the paper and commented on the text.

\section{Additional information}

Supplementary Information accompanies this paper at http://www.nature.com/ naturecommunications

Competing financial interests: The authors declare no competing financial interests.

Reprints and permission information is available online at http://npg.nature.com/ reprintsandpermissions/

How to cite this article: Zhao, W. et al. Multi-localization transport behaviour in bulk thermoelectric materials. Nat. Commun. 6:6197 doi: 10.1038/ncomms7197 (2015).

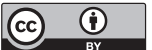

This work is licensed under a Creative Commons Attribution 4.0 International License. The images or other third party material in this article are included in the article's Creative Commons license, unless indicated otherwise in the credit line; if the material is not included under the Creative Commons license, users will need to obtain permission from the license holder to reproduce the material. To view a copy of this license, visit http://creativecommons.org/licenses/by/4.0/ 